\title{
Editorial
}

\section{Animal Models of Human Pathology 2016}

\author{
Monica Fedele, ${ }^{1}$ Oreste Gualillo, ${ }^{2}$ and Andrea Vecchione ${ }^{3,4}$ \\ ${ }^{1}$ Istituto per l'Endocrinologia e l'Oncologia Sperimentale (IEOS), CNR, 80131 Naples, Italy \\ ${ }^{2}$ Santiago University Clinical Hospital, Instituto de Investigación Sanitaria de Santiago (IDIS), 15706 Santiago de Compostela, Spain \\ ${ }^{3}$ Human Cancer Genetics Program/CCC, The Ohio State University, Columbus, OH 43210, USA \\ ${ }^{4}$ Department of Clinical and Molecular Medicine, Sapienza University of Rome, 00100 Rome, Italy
}

Correspondence should be addressed to Monica Fedele; mfedele@unina.it

Received 29 November 2016; Accepted 29 November 2016

Copyright (C) 2016 Monica Fedele et al. This is an open access article distributed under the Creative Commons Attribution License, which permits unrestricted use, distribution, and reproduction in any medium, provided the original work is properly cited.

Biomedical research is a complicated process that needs tools capable of mimicking the human body level of complexity. Despite increasingly sophisticated in vitro model systems, animal models remain irreplaceable allies for the study of human disease. The paraphrase "in vivo veritas" is today more relevant than ever, so that the scientific community is doing its best to develop animal models ever closer to man. In the last few years, with the development of humanized mice, increasingly optimized to mimic human immune system and allow the efficient engraftment of human cells, we entered in a new era of translational research, where animal models are very close to humans and play a crucial role in the development of new therapeutic strategies for human pathologies.

In this new collection of articles (15 research articles and 5 reviews) focused on animal models of human pathology, we have gathered interesting evidences of how, through the use of animal models, we can understand, ever more closely, the pathogenic mechanisms of a human disease. Moreover, we have learned about new animal models and new therapeutic strategies for different human diseases. Most of the animal models used are small rodents, such as mice and rats, but you will also read about rabbits, dogs, goats, pigs, zebrafish, chick embryos, ferrets, and primates.

C. Daly, M. Fusellier, and respective coauthors faced the problem of the intervertebral disc degeneration, from pathophysiology to the clinic. Other pathologies approached by animal models in this special issue include cerebral and kidney ischemic events, dialed by I. Novitzky, S.-J. Chang, A. Lazaro, and respective coauthors; diabetes mellitus, with the contribution of M. Du et al.; inflammation, addressed by W.-R. Lin, S. Biesmans, and respective coauthors; cystic fibrosis, contributed by G. M. Lavelle et al.; and obesity, with the article by K. Nakadate et al. that continue their recent studies carried out on this pathology and its relationship with metabolic syndrome.

Among the contributions to cancer, three articles dial with colorectal cancer, optimizing patient-derived xenograft models (M. Gock et al.), studying the protective effect of Vitamin A (I. Okayasu et al.), and reviewing the role of deficiencies of DNA repair mechanisms (M. A. Mcilhatton et al.) through mouse models. In this field you will also read the timely and updated reviews of D. Rea et al. about prostate cancer and M. M. Stei et al. about uveal melanoma.

New animal models of human disease are addressed by S. H. Abdul Aziz et al. that describe the development of a rat model of gestational diabetes mellitus based on the combination of high fat sucrose diet with streptozotocin and nicotinamide STZ; J. Bogomolovas et al. that develop a novel murine model of parvovirus associated dilated cardiomyopathy; and V. Sala et al., with their new transgenic mouse model of heart failure and cardiac cachexia.

Lastly, the antifatigue effects of an asiatic mushroom and the protective effect of erythropoietin treatment in animals exposed to ventricular fibrillation were studied by Q. Li et al. and A. Faa et al., respectively.

\section{Acknowledgments}

We thank all authors for their contribution and the expert reviewers for their critical analysis of the manuscripts, hoping 
that, once again, we provided the readership with an interesting overview of what it is going on in the biomedical field thanks to the lessons learned from animal models.

Monica Fedele

Oreste Gualillo

Andrea Vecchione 


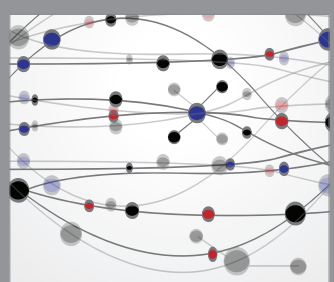

The Scientific World Journal
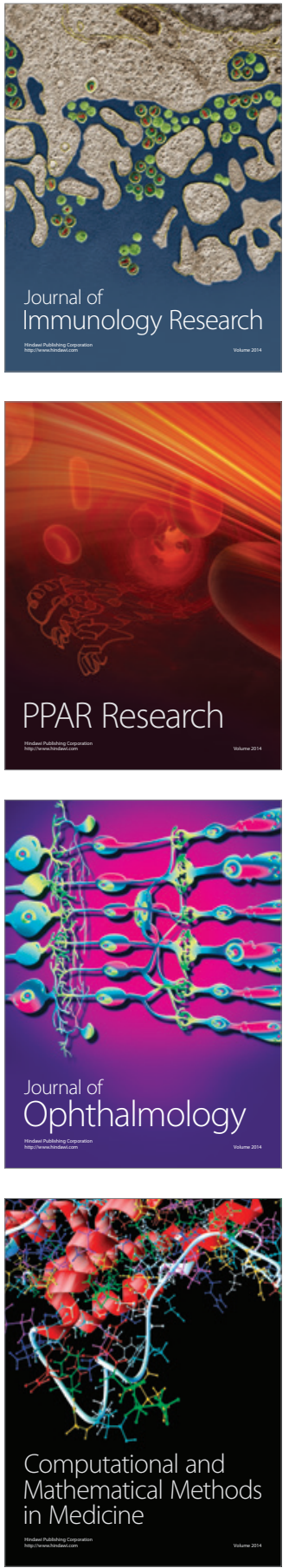

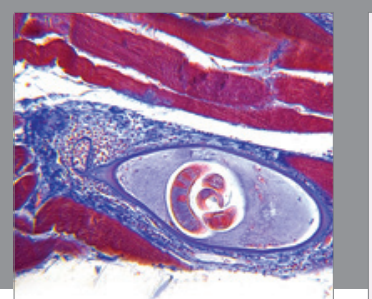

Gastroenterology Research and Practice

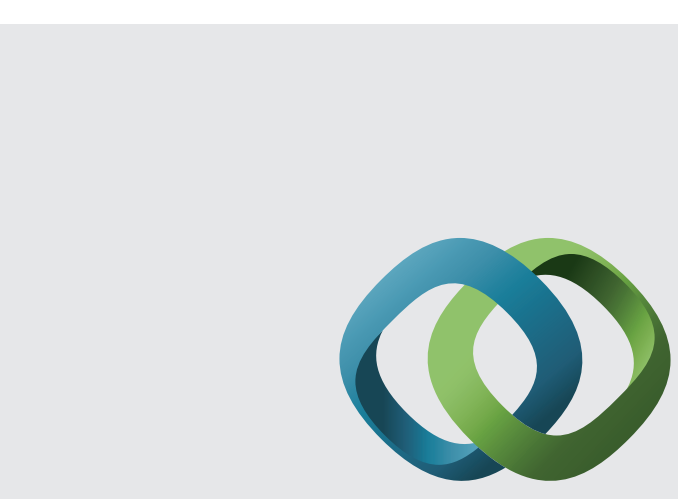

\section{Hindawi}

Submit your manuscripts at

http://www.hindawi.com
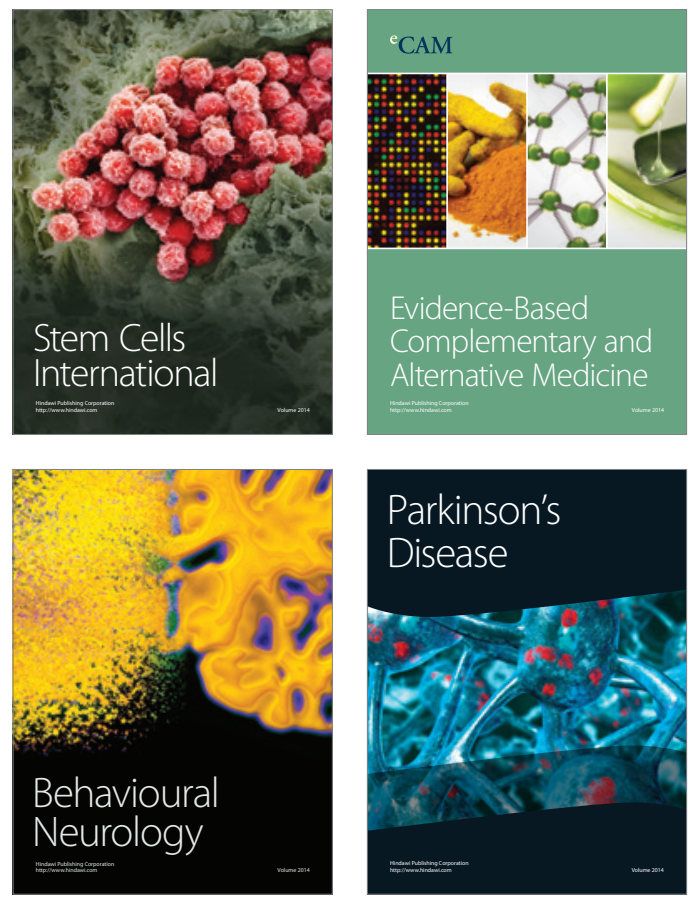
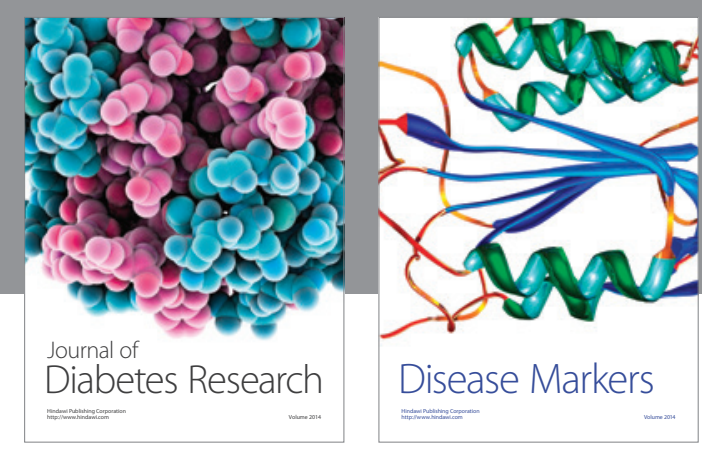

Disease Markers
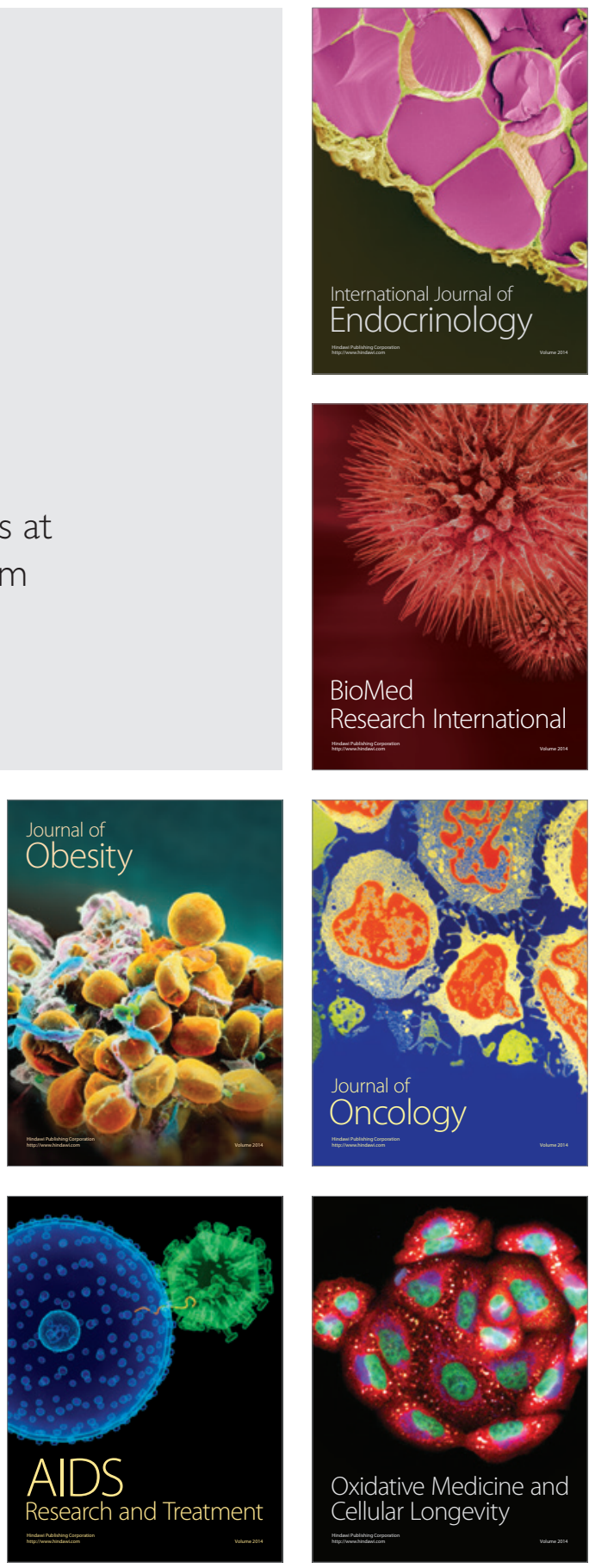